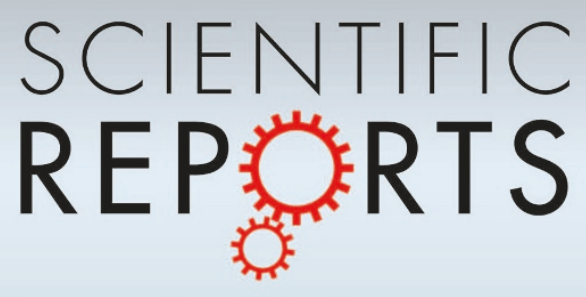

OPEN

SUBJECT AREAS:

SPINTRONICS

MAGNETIC PROPERTIES AND MATERIALS

Received 1 May 2014

Accepted

17 September 2014

Published

8 October 2014

Correspondence and requests for materials should be addressed to C.-Y.Y. (cyyou@inha. ac.kr) or M.-H.J. (mhjung@sogang.ac.

\title{
Spin-orbit torque in a bulk perpendicular magnetic anisotropy $\mathrm{Pd} / \mathrm{FePd} / \mathrm{MgO}$ system
}

\author{
Hwang-Rae Lee', Kyujoon Lee ${ }^{2}$, Jaehun Cho' , Young-Ha Choi' ${ }^{2}$ Chun-Yeol You' , Myung-Hwa Jung ${ }^{2}$, \\ Frédéric Bonell ${ }^{3,4}$, Yoichi Shiota ${ }^{3,4}$, Shinji Miwa ${ }^{3,4}$ \& Yoshishige Suzuki ${ }^{3,4}$
}

\begin{abstract}
'Department of Physics, Inha University, Incheon 402-751, Korea, ${ }^{2}$ Department of Physics, Sogang University, Seoul 121-742, Korea, ${ }^{3}$ Graduate School of Engineering Science, Osaka University, Osaka 560-853 1, Japan, ${ }^{4}$ CREST, Japan Science Technology Agency, Saitama 332-0012, Japan.
\end{abstract}

Spin-orbit torques, including the Rashba and spin Hall effects, have been widely observed and investigated in various systems. Since interesting spin-orbit torque (SOT) arises at the interface between heavy nonmagnetic metals and ferromagnetic metals, most studies have focused on the ultra-thin ferromagnetic layer with interface perpendicular magnetic anisotropy. Here, we measured the effective longitudinal and transverse fields of bulk perpendicular magnetic anisotropy Pd/FePd (1.54 to $2.43 \mathrm{~nm}$ )/MgO systems using harmonic methods with careful correction procedures. We found that in our range of thicknesses, the effective longitudinal and transverse fields are five to ten times larger than those reported in interface perpendicular magnetic anisotropy systems. The observed magnitude and thickness dependence of the effective fields suggest that the SOT do not have a purely interfacial origin in our samples.

\footnotetext{
S ince the discovery of spin transfer torque (STT), the method of manipulation of the magnetization direction has been shifted from the Oersted field to the spin current ${ }^{1,2}$. STT has been widely studied, and it is a building block of next-generation non-volatile random access memory. More recently, another magnetization manipulation method has been reported for a few atomic magnetic layers sandwiched with heavy metal and oxide layers ${ }^{3-8}$. The charge current passing through a heavy nonmagnetic metal layer produces effective fields by two known mechanisms: the Rashba effect and/or the spin Hall effect (SHE). Both phenomena are related to strong spin-orbit coupling, so they are called the spin-orbit torque (SOT) effect. It has been reported that SOT can alter the spin dynamics in an ultra-thin ferromagnetic layer through the effective fields ${ }^{9}$, and move the domain wall much faster than by STT only. Also, the domain wall motion direction can be opposite to the electron flow ${ }^{10}$. At the early stage of the study, it has been known that the Rashba effect generates a transverse effective field (or field-like term), while the SHE produces a longitudinal effective field (or damping-like term). However, refined models recently showed that both mechanisms generate both transverse and longitudinal effective fields ${ }^{11}$; thus, distinguishing between them remains challenging issues. Using SOT combines several advantages compared to the STT in spin valves. For example, the SHE uses only pure spin current that is generated in nonmagnetic heavy metals to manipulate the magnetization of the magnetic layer, and requires only a single magnetic layer. In contrast, a second reference ferromagnetic layer is inevitable in the STT technique, and large currents have to be set across a tunnel junction. The efficiency of the SOT is superior to that of the STT.

It is believed that the Rashba effect is generated in the conduction electrons at the interface between the nonmagnetic heavy metal and the magnetic layer by strong spin-orbit coupling. Conduction electrons under the finite potential gradient feel the effective magnetic field due to the strong spin-orbit coupling, as described by special relativity. And, the conduction electrons are strongly coupled with the localized $d$-electrons. Therefore, the spin motions of the localized $d$-electrons are also affected by the Rashba effective field ${ }^{12}$. On the other hand, the SHE is a diffusive spin current from the adjacent heavy nonmagnetic metal volume to the magnetic layer. The injected spin currents rapidly decay due to Fermi surface averaging, similar to STT in a metallic spin valve structure $^{13-16}$. Therefore, both SOTs can be handled as interface effective fields.

Most studies have been performed for only a few atomic magnetic layers having interfacial perpendicular magnetic anisotropy (PMA), such as $\mathrm{Co}$ or $\mathrm{CoFeB}$, which are sandwiched between nonmagnetic heavy metals $(\mathrm{Pt}$, $\mathrm{Ta}, \mathrm{W})$ and oxide layers $\left(\mathrm{AlO}_{\mathrm{x}}, \mathrm{MgO}\right)$. The interfacial PMA system maintains its out-of-plane easy axis only for limited thickness ranges. For example, Kim et al. investigated a Ta/CoFeB/MgO system with a 0.9 to $1.3 \mathrm{~nm}$-thick
} 
CoFeB layer ${ }^{7}$. Due to the interfacial nature of PMA, it shows an inverse proportionality to the ferromagnetic layer thickness. Fan et al. studied in-plane anisotropy materials of Py (10 $\mathrm{nm}$ thickness) by measuring transport properties ${ }^{17}$, and the crossover from the perpendicular anisotropy of thin $\mathrm{CoFeB}$ to the in-plane anisotropy of thick $\mathrm{CoFeB}$, by measuring the magneto-optical Kerr effect. They found that the longitudinal effective field is inversely proportional to the thickness of the magnetic layer, and that the transverse effective field decreases rapidly when the magnetic layer is thicker than $1 \mathrm{~nm}^{18}$. Jamali et al. investigated a $[\mathrm{Co}(0.2 \mathrm{~nm}) / \mathrm{Pd}(0.7 \mathrm{~nm})]$ multilayer system and reported huge effective fields for the longitudinal $\left(11.7 \mathrm{Oe} / 10^{10} \mathrm{~A} / \mathrm{m}^{2}\right)$ and transverse $\left(50.25 \mathrm{Oe} / 10^{10} \mathrm{~A} / \mathrm{m}^{2}\right)$ directions. This suggests that the Rashba effective field is important despite the symmetric structure of their samples ${ }^{19}$. More recently, Qiu et al. reported temperature and angular dependences of SOT in a $\mathrm{Ta} /$ $\mathrm{CoFeB} / \mathrm{MgO}$ system ${ }^{20}$. They found that the transverse field decreased with decreasing temperature, whereas the longitudinal field showed weak temperature dependence. This implies that the physical origins of two effective fields may different. So far, reported results show that the purely interfacial nature of SOT is questionable.

In this study, we examined SOT in a Pd/FePd (1.54 to $2.43 \mathrm{~nm}) /$ $\mathrm{MgO}$ system with a bulk PMA due to the $\mathrm{L} 1_{0}$ crystalline structure of $\mathrm{FePd}^{21}$. By virtue of the bulk $\mathrm{L}_{0}$ crystalline PMA, we could extend the SOT research to much thicker ferromagnetic layer systems compared to other groups. With careful analysis by considering the planar Hall effect and Oersted field contributions, we found that the longitudinal effective field is five to ten times stronger than, or comparable to, that of a $\mathrm{Ta} / \mathrm{CoFeB} / \mathrm{MgO}$, when we considered the ferromagnetic layer thickness ${ }^{7,20}$. The experimentally observed thickness dependencies of the transverse and longitudinal effective fields arise a question about the interface origin of SOT.

\section{Results}

Measurement of the longitudinal and transverse effective fields. Since the origins of the effective transverse and longitudinal fields are not easily distinguishable, we focused on systematic measurements of the effective longitudinal and transverse fields in $\mathrm{Pd} / \mathrm{Ll}_{0}-\mathrm{FePd}$ (1.54 to $2.43 \mathrm{~nm}$ )/MgO wedge samples, as shown in Fig. 1a. In this geometry, the applied current produces two effects. SHE separates the charge current to up- and down-spin current, as shown in Fig. 1b, and it mainly leads to the longitudinal effective field $\left(H_{L}\right)$. The potential gradient at the interface generates the Rashba effect, which causes the transverse effective field $\left(H_{T}\right)$, as shown in Fig. 1b. However, the Rashba effect (SHE) can also generate the longitudinal (transverse) fields ${ }^{11}$.

The measurement geometry for the $2 \omega$ method with the coordinate system is shown in Figs. $1 \mathrm{c}$ and $\mathrm{d}^{7,22,23}$. A $100 \mu \mathrm{m} \times 10 \mu \mathrm{m}$ wire was connected to the two electric pads with two pairs of Hall bar pads. The measured Hall signals are comprised of the anomalous Hall effect (AHE) and the planar Hall effect (PHE):

$$
V_{\mathrm{Hall}}=\frac{V_{\mathrm{AHE}}}{2} \cos \theta+\frac{V_{\mathrm{PHE}}}{2} \sin ^{2} \theta \sin 2 \phi .
$$

Here, $V_{\mathrm{AHE}}=I_{0} R_{\mathrm{AHE}}$ and $V_{\mathrm{PHE}}=I_{0} R_{\mathrm{PHE}}$ are the Hall voltages of the $\mathrm{AHE}$ and PHE and $R_{\mathrm{AHE}}$ and $R_{\mathrm{PHE}}$ are corresponding Hall resistances, respectively. The applied alternating current (AC) current is given by $I(t)=I_{0} \sin \omega t$. The polar angle $\theta$ and azimuthal angle $\phi$ of the magnetization direction are defined in the inset of Fig. 1b, and they are determined mainly by the external magnetic field and the system anisotropy energy. However, they oscillate with a modulation frequency of $\omega$ due to the external AC effective field. Therefore, the measured Hall voltage can be expressed as

$$
V_{\text {Hall }}=V_{d c}+V_{\omega} \sin \omega t+V_{2 \omega} \cos 2 \omega t+\cdots .
$$

It is known that the effective longitudinal $\left(H_{L}, y\right.$-axis $)$ and transverse $\left(H_{T}, x\right.$-axis) fields can be determined from the ratio between the slope of the out-of-phase second harmonics of the Hall voltage and the curvature of the in-phase first harmonics, as follows ${ }^{7,21}$ :

$$
H_{\mathrm{L}, \mathrm{T}}=-2 \frac{\partial V_{2 \omega}}{\partial H_{\mathrm{ext}, y / x}} / \frac{\partial^{2} V_{\omega}}{\partial H_{\mathrm{ext}, y / x^{2}}},
$$

where $H_{\text {ext }, x / y}$ are the external applied transverse $(x)$ and longitudinal $(y)$ fields during the AHE measurements, and $V_{\omega}$ and $V_{2 \omega}$ are the first and second harmonics of the AHE voltages, respectively. Equation (3) is valid only when the PHE contribution is ignored. However, the PHE cannot be generally ignored and must be carefully considered. With valid approximations, we obtained the following equations (see Sec. S2 in Supplementary Information):

$$
\begin{gathered}
H_{L, \text { meas } \pm}=-2 \frac{\partial V_{2 \omega, L \pm}}{\partial H_{\mathrm{ext}, y}} / \frac{\partial^{2} V_{\omega, L \pm}}{\partial H_{\mathrm{ext}, y}{ }^{2}}= \pm\left(H_{L}-2 \chi H_{T}\right), \\
H_{T, \text { meas } \pm}=-2 \frac{\partial V_{2 \omega, T \pm}}{\partial H_{\mathrm{ext}, x}} / \frac{\partial^{2} V_{\omega, T \pm}}{\partial H_{\mathrm{ext}, x}{ }^{2}}=\left(H_{T}-2 \chi H_{L}\right) .
\end{gathered}
$$

where $\chi=R_{\mathrm{PHE}} / R_{\mathrm{AHE}}$ is the resistance ratio between $\mathrm{AHE}$ and $\mathrm{PHE}$, $H_{L, \text { meas }}$ and $H_{T, \text { meas }}$ are the experimentally measured results from the first and second harmonic signals, respectively, and the \pm sign represents up and down magnetization $\left( \pm M_{z}\right)$, respectively. $H_{L}$ and $H_{T}$ represent the real effective field that we want to determine in the present experiments. Equations (4) and (5) describe the effective fields obtained by Kim et al. ${ }^{7}$, and they are actually mixed signals of the longitudinal and transverse effective fields whenever the PHE is nonzero. From Eqs. (4) and (5), $H_{L}$ and $H_{T}$ can be readily extracted if $\chi$ is known, as follows ${ }^{22}$ :

$$
\begin{aligned}
& H_{L}=\frac{H_{L, \text { meas } \pm} \pm 2 \chi H_{T, \text { meas } \pm}}{1-4 \chi^{2}}, \\
& H_{T}=\frac{H_{T, \text { meas } \pm} \pm 2 \chi H_{L, \text { meas } \pm}}{1-4 \chi^{2}},
\end{aligned}
$$

where the \pm sign represents the up and down magnetizations. With corrected expressions, one can separate the PHE contribution in the measurement signals. We note that if $\chi$ is small, the PHE contribution is negligible. More details are given in the Supplementary Information (S2).

Experimental results, and other contributions to the longitudinal and transverse effective fields. Figures $2 \mathrm{a}-\mathrm{f}$ show typical experimental results in the present study for a $1.54 \mathrm{~nm}$ FePd sample. Figure 2a shows an AHE hysteresis loop. The Hall voltage was measured with the external field in the $z$-direction $\left(H_{\mathrm{z}}\right)$, and the detecting current was small enough so that the effective field due to the SOT is negligible. Figure $2 \mathrm{~b}$ shows the first harmonics of Hall voltage $V_{\omega}$ with the transverse and longitudinal external fields $\left(H_{\mathrm{x}}\right.$ and $\left.H_{\mathrm{y}}\right)$. With moderate field strength, the first harmonic signals are approximated by a parabola to the external field, as shown in Fig. 2 b. The small difference in curvatures between the transverse and longitudinal $V_{\omega}$ are due to the finite in-plane shape anisotropy. We applied a moderate strength field to keep $\theta \ll 1$. More details regarding $V_{\omega}$ are given in Supplementary Information (S2). Figures $2 \mathrm{~d}$ and e are the out-of-phase transverse and longitudinal $V_{2 \omega}$ for the up and down magnetizations with corresponding external in-plane fields. Here, the transverse (longitudinal) $V_{2 \omega}$ is odd (even) with respect to the magnetization inversion due to the symmetry of the given problem. Note that the $V_{2 \omega}$ signals are much more scattered than those reported by other groups $s^{7,17,18,23}$. The main reason for the scattered signals is the $18.5 \mathrm{~nm}$-thick Pd underlayer, which is necessary to promote better $\mathrm{L} 1_{0}$ crystal structure of the FePd layer. A large shunt current flowed through the thick Pd underlayer, and only a small part of the current contributed to the AHE signal. 
a

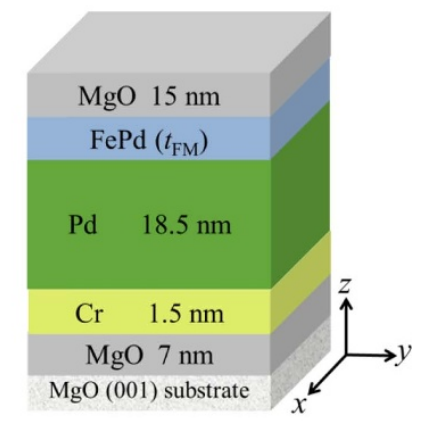

C

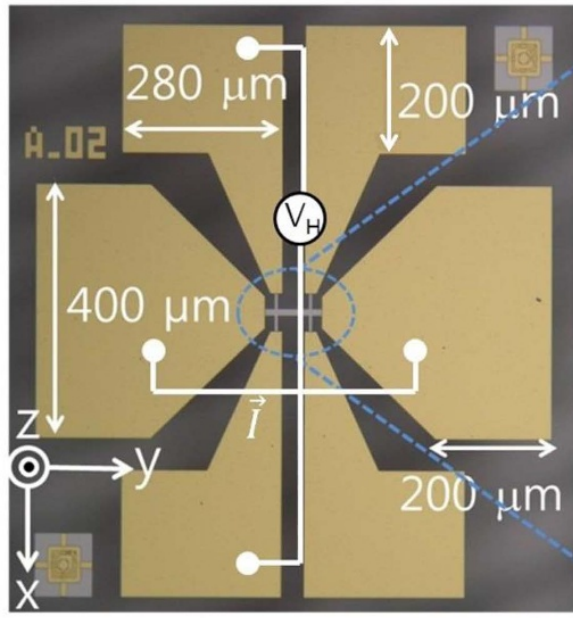

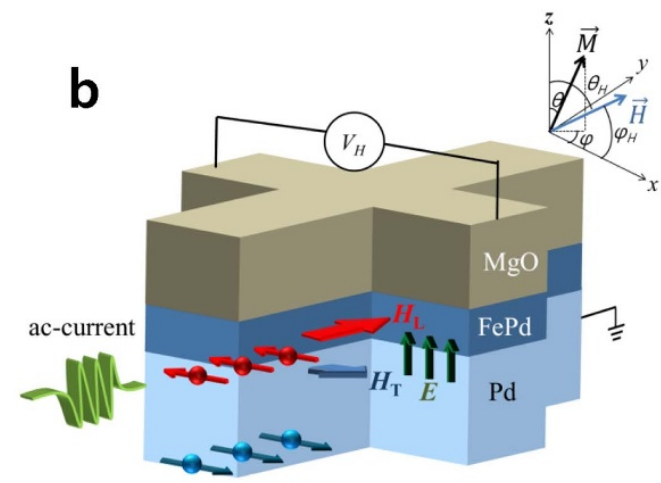

d

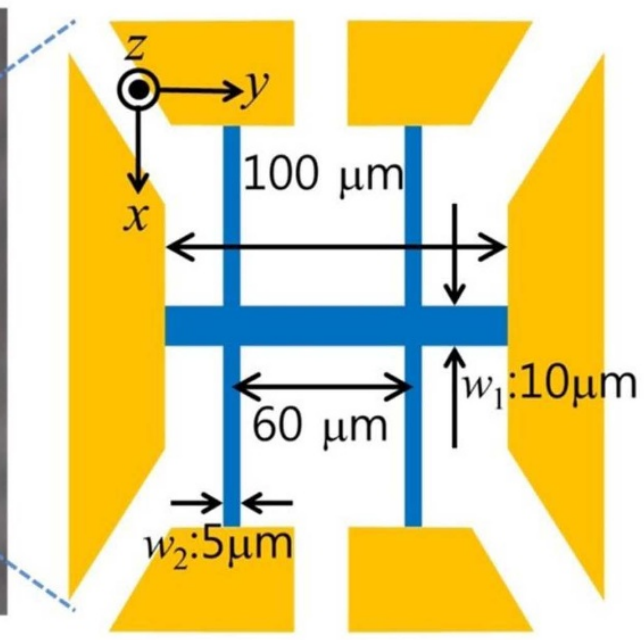

Figure 1 | Schematic sketches of sample structure, Hall bar geometry with SOT, and patterns. (a) Sample structure of $\mathrm{MgO}(001) / \mathrm{MgO}(7 \mathrm{~nm}) / \mathrm{Cr}$ $(1.5 \mathrm{~nm}) / \mathrm{Pd}(18.5 \mathrm{~nm}) / \mathrm{FePd}\left(t_{F M}\right) / \mathrm{MgO}(15 \mathrm{~nm})$. The thickness of FePd was varied from 1.54 to $2.43 \mathrm{~nm}$. (b) Hall bar geometry and measurement setup. The AC current flows in the $+y$-direction, the SHE provides spin current to the $\pm z$-directions, and the finite potential gradient is developed at the $\mathrm{Pd} / \mathrm{FePd}$ interface. The longitudinal and transverse effective fields $\left(H_{L}\right.$ and $\left.H_{T}\right)$ are shown, and the definition of the polar and azimuthal angles of the magnetization and external fields are shown with the corresponding coordinate system. (c) Photos of the Hall bar patterns with electrodes. (d) More details of the Hall bar geometry with wire dimensions.

Despite the scattered signals, it is sufficient to determine the slope of $V_{2 \omega}$, as shown in Figs. $2 \mathrm{~d}$ and $\mathrm{e}$.

The PHE measurement is shown in Fig. 2c. In order to measure the PHE, we applied a $\pm 1.0 \mathrm{kOe}$ longitudinal field (that is the maximum field of our apparatus). It is sufficient to lay the magnetization down on the in-plane for the thinnest FePd $(1.54 \mathrm{~nm})$ sample, whose perpendicular magnetic anisotropy is the smallest. While keeping the $\pm 1.0 \mathrm{kOe}$ longitudinal field, we added a small transverse field $(\sim 100$ Oe $)$ to detect the planar Hall signal. Since the in-plane anisotropy field $H_{\text {in }}=\left(N_{x}-N_{y}\right) M_{s} \sim 100$ Oe was much smaller than the external longitudinal field ( $\pm 1.0 \mathrm{kOe}$ ), we ignored the effect of the in-plane anisotropy, and the azimuthal angle $\phi$ of the magnetization is almost the same as the in-plane field angle $\phi_{H}=\pi / 2-\arctan \left(H_{x} /\right.$ $\left.H_{y}\right)$. With these assumptions, we extracted $V_{P H E}$ from Eq. (1), and then determined $V_{\mathrm{PHE}}=6.01 \pm 0.05 \mu \mathrm{V}$ and $V_{\mathrm{AHE}}=25.4 \mu \mathrm{V}$ from Figs. $2 \mathrm{a}$ and c. This means that the PHE contribution, $\chi=0.237$, is not negligible in the present experiments. Thus, we used $\chi$ for a careful analysis of our experimental data using Eqs. (6) and (7).

Another important contribution in the measured Hall voltages is the anomalous Nernst-Ettingshausen (ANE) effect due to inhomogeneous heating across the Hall bar geometry. This inhomogeneous heat distribution is unavoidable in the harmonic measurement experiments. The ANE contribution is described by Garello et al. ${ }^{24}$. We followed their method, but we could not find any noticeable ANE signals, as shown in Fig. $2 \mathrm{f}$. The scales of the $y$-axes in Figs. $2 \mathrm{~d}-\mathrm{f}$ are the same for easy comparison. Compared to the Hall contribution, the ANE contribution can be ignored in our experiments. The negligible ANE may come from the $18.5 \mathrm{~nm}$-thick Pd underlayer. Compared to other experiments, we used a rather thick Pd underlayer to promote better $\mathrm{L}_{0}$ crystal structure in the FePd layer. Such a thick Pd underlayer acts as a good heat buffer, and ensures better uniform heating or a negligible temperature gradient. Although the $V_{2 \omega}$ data are very scattered because of the thick Pd underlayer, the thick Pd has the advantage of suppressing the unwanted contribution of the ANE.

In addition, the Joule heating effect must be addressed. The details of the Joule heating effect are discussed in the Supplementary Information (S4). In summary, the Joule heating due to the applied current is about 40 to $50 \mathrm{~K}$ for a maximum current density of $2.7 \times$ $10^{11} \mathrm{~A} / \mathrm{m}^{2}$. If the temperature increment alters the magnetic properties, it is clear that the behavior of the AHE with respect to the applied voltage must deviate from the linear. In addition, the curvature of $V_{\omega}$, which is related to the effective perpendicular magnetic anisotropy energy, must be changed with the applied voltage. However, the AHE signal of the thinnest FePd is linear with respect to the applied AC voltage until reaching the maximum applied current density (Fig. S5a in the Supplementary Information), and the $V_{\omega}$ data with various voltages collapse to the single curve (Fig. S5b). These results indicate that the magnetic properties of the sample are not noticeably changed by the Joule heating despite the temper- 

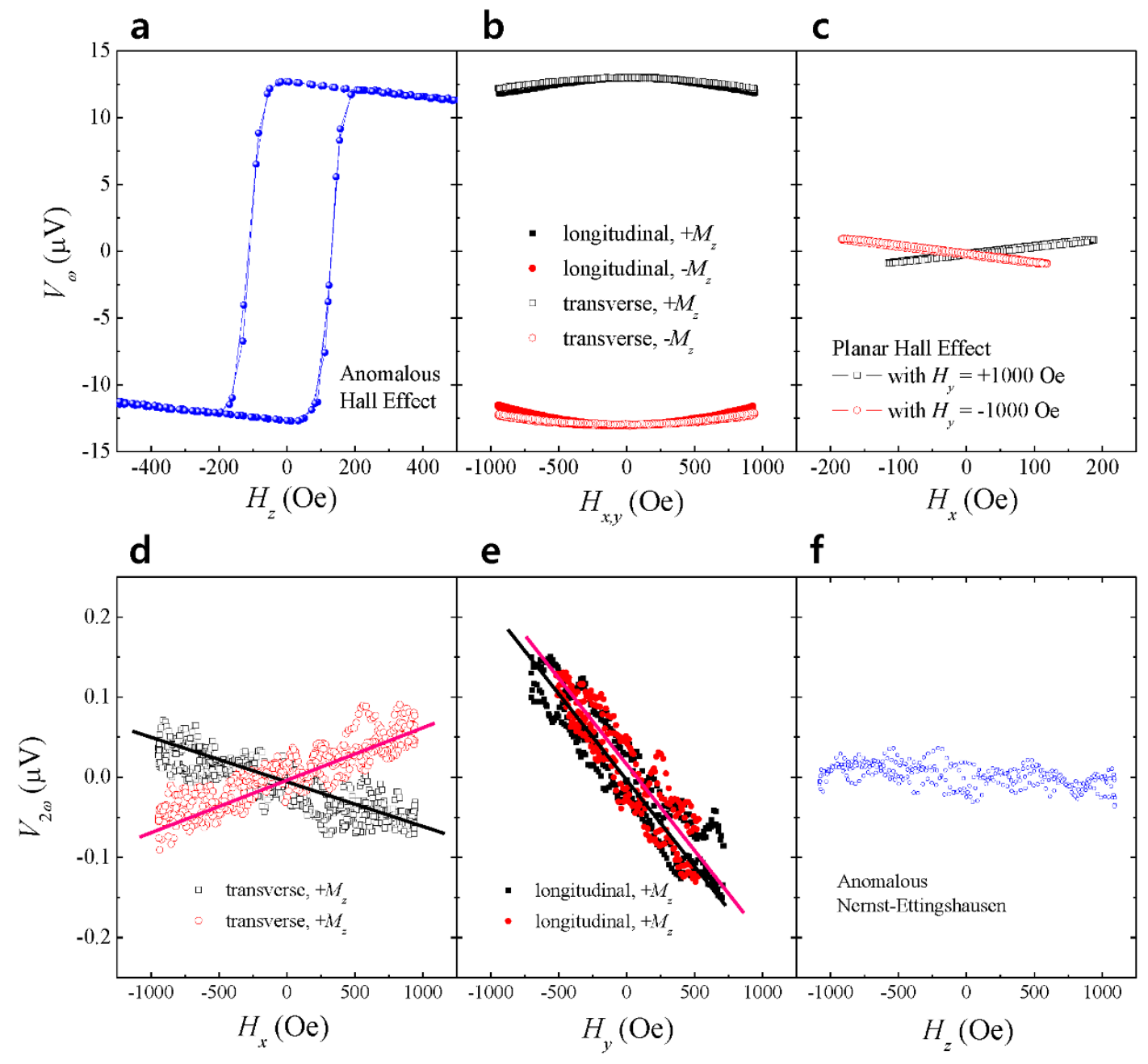

Figure $2 \mid$ Experimentally measured data of $1.54 \mathbf{n m}$-thick FePd. (a) The anomalous Hall effect (AHE) hysteresis loop by the AC detection method with $H_{z}$. (b) $V_{\omega}$ signals for the longitudinal (transverse) in-plane external field $H_{y}\left(H_{x}\right)$, respectively. The AHE signal represents the cos $\theta \operatorname{component}$ of the magnetization. (c) Planar Hall effect (PHE) measurements. The magnetization is aligned with the $\pm y$-axis with $\pm 1.0 \mathrm{kOe} H_{y}$, and an additional small $H_{x}$ is applied to determine $V_{P H E}$ (d) Out-of-phase second harmonics $V_{2 \omega}$ with the transverse external field $H_{x}$ for up and down magnetization.

(e) Out-of-phase second harmonics $V_{2 \omega}$ with the longitudinal external field $H_{y}$ for up and down magnetization. (f) Anomalous Nernst-Ettingshausen (ANE) effect measurement. All $y$-axes of (a), (b), and (c), and (d), (e), and (f), respectively, use the same scale for easy comparisons.

ature increment of $\sim 50 \mathrm{~K}$. Based on these observations, we suggest that the Joule heating effect is not important in the present experiments.

Finally, the Oersted field contribution is also important in our sample structure. Since the nonmagnetic layer composed of $1.5 \mathrm{~nm}$-thick $\mathrm{Cr}$ and $18.5 \mathrm{~nm}$-thick Pd is thicker than that in the structures of other groups, the total current is larger for a given current density. The estimated Oersted field is $H_{\mathrm{oe}} \sim J t_{\mathrm{Pd}+\mathrm{Cr}} / 2 \sim$ 1.25 Oe for $10^{10} \mathrm{~A} / \mathrm{m}^{2}$. This value is comparable to the strength for the effective transverse field, and is almost ten times larger than that reported by Kim et al. ${ }^{7}$ It is also due to the thicker Pd underlayer. Importantly, the Oersted field is positive in our experimental geometry, while the measured transverse effective field is always negative. This means that the Oersted field always reduces the magnitude of the transverse effective field due to the SOT. We will correct the Oersted field contribution in the final results.

Thickness dependence of the SOT effective fields. Figure 3 a shows the measured longitudinal and transverse effective fields ( $-H_{L \text {,measured }}$ and $\left.H_{T \text {,measured }}\right)$ determined using Eqs. (4) and (5), respectively, which are mixtures of the pure longitudinal and transverse effective fields. We plot $-H_{L \text {,measured }}$ and $H_{T \text {,measured }}$ in Fig. 3a for $t_{\mathrm{FePd}}=1.54,1.73,2.13$, and $2.43 \mathrm{~nm}$, together with linear fits. The applied AC voltage was converted to the corresponding current density $J$. As previously discussed, the effective field does not deviate from linear behavior, which implies that Joule heating is not significant in our experiments. We note that the signs of $H_{L}$ are negative (positive) for up (down) magnetizations, and the signs of $H_{T}$ are always negative for up and down magnetizations (Figs. $2 \mathrm{~d}$ and $\mathrm{e}$, and Eqs. (4) and (5)). Therefore, we plotted $-\left(H_{L,+}-H_{L,-}\right) / 2$ and $\left(H_{T,+}+H_{T,-}\right) / 2$ in Fig. $3 \mathrm{a}$ for easy comparisons. With these measured effective fields and the ratio of PHE and AHE $(\chi=$ 0.237 ), we corrected the effective fields using Eqs. (6) and (7). Due to the small value of $1 /\left(1-4 \chi^{2}\right)=1.29$ in Eqs. (6) and (7), and mixed contributions of the pure longitudinal and transverse fields, the corrected effective fields are somewhat larger than the measured ones. Especially, the corrected transverse effective fields are much larger than the measured fields. For example, the measured transverse fields of $1.54 \mathrm{~nm}$ are the smallest among the series (Fig. 3a), but they turn to be the largest after correction (Fig. 3b). Since the measured transverse field is equal to $H_{T \text {,meas } \pm}=H_{T}-$ $2 \chi H_{L}$, where $H_{T}$ and $H_{L}<0,\left|H_{T \text {,meas } \pm}\right|$ is smaller than $\left|H_{T}\right|$. Therefore, careful determination of the signs is crucial.

The corrected transverse fields in Fig. 3 still include the Oersted field. Therefore, an additional conversion, $H_{T, \mathrm{SOT}}=H_{T \text {,corrected }}-$ 
a

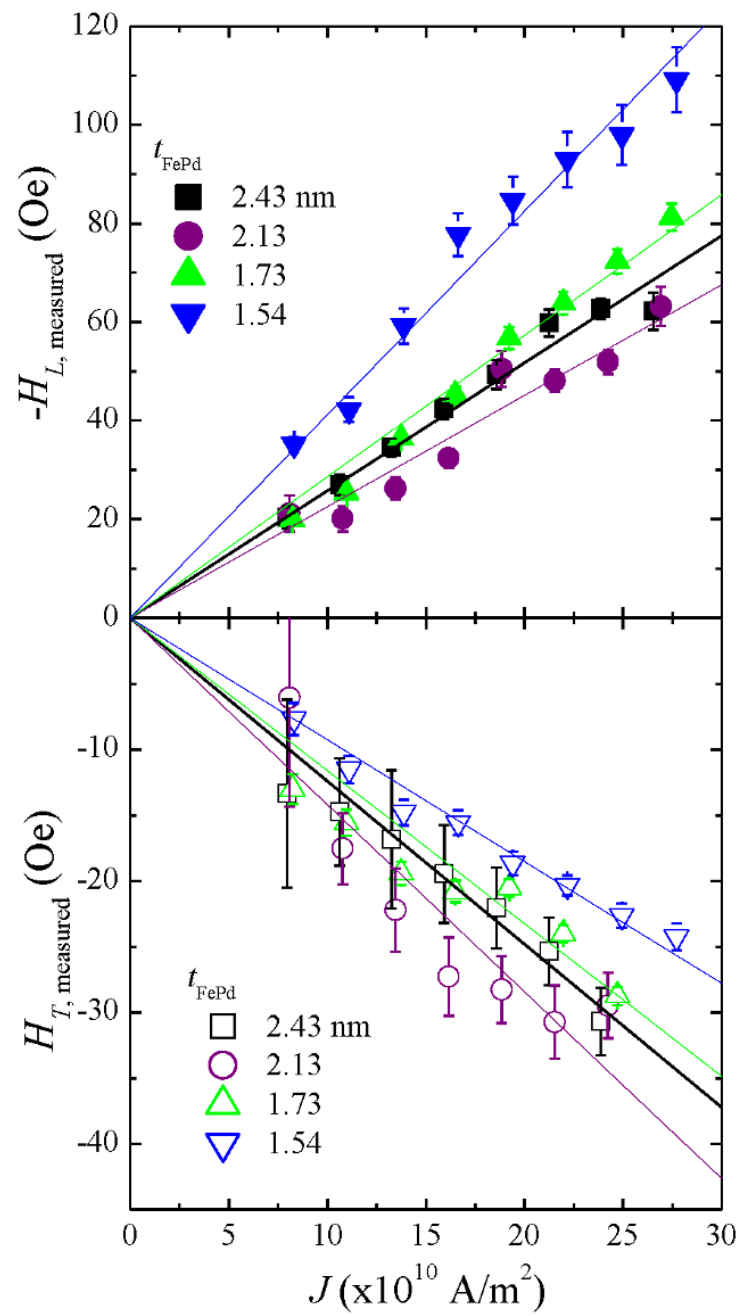

b

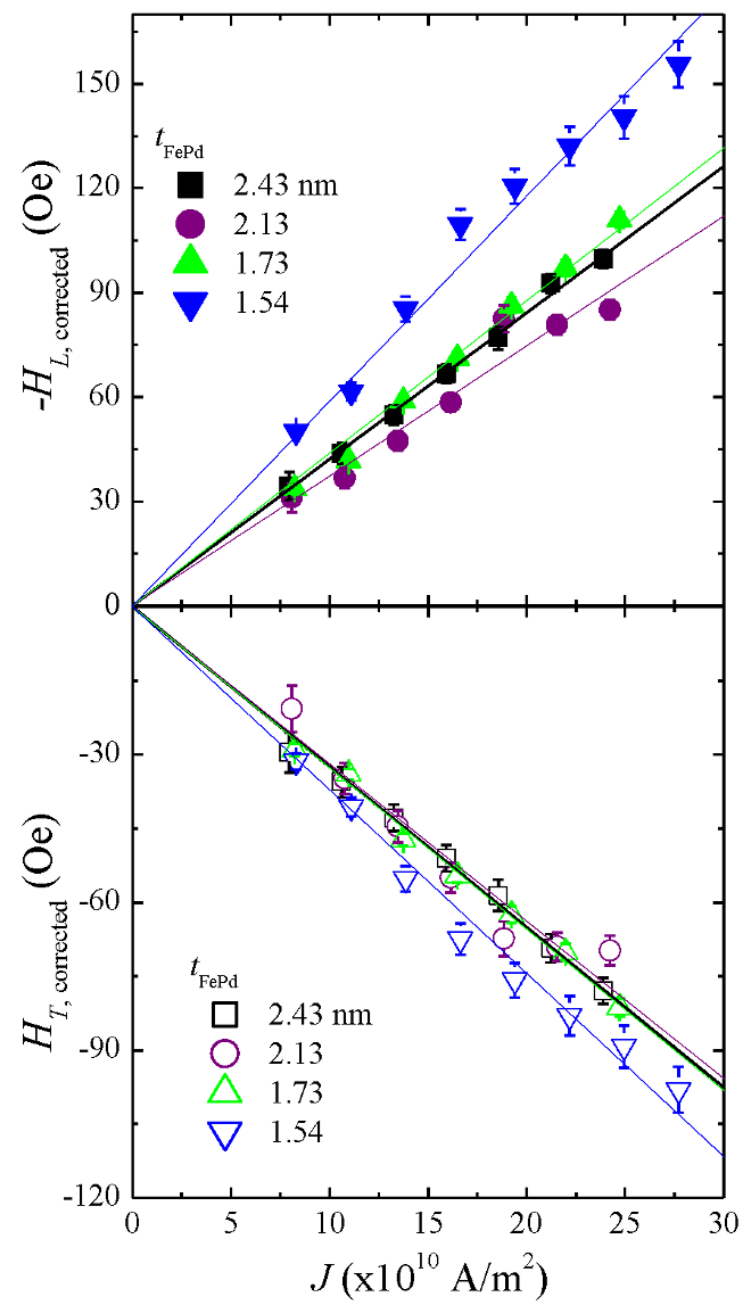

Figure 3 Longitudinal and transverse effective fields. (a) The measured effective fields, determined using Eqs. (4) and (5) for 1.54 to 2.43 nm-thick FePd. The signs of $H_{L, \text { measured }}$ and $H_{T \text {,measured }}$ are negative, and the solid line represents linear fittings. (b) Corrected effective fields based on Eqs. (6) and (7) with $\chi=0.237$.

$H_{\text {Oersted, }}$, is required to obtain the pure effective field, again with the proper sign considerations $\left(H_{T \text {,measured }}<0\right.$ and $\left.H_{\text {Oersted }}>0\right)$. We compare these fully corrected effective fields with the results of other groups in Fig. 4. We scaled the effective fields from the literatures s,9,18,20,24 $^{70}$ a current density of $10^{10} \mathrm{~A} / \mathrm{m}^{2}$ (assuming linearity with current), and plotted them as a function of the inverse ferromagnetic layer thickness, $1 / t_{\mathrm{FM}}$. For easy comparison, we took the absolute values of each effective field. Qiu et al. ${ }^{20}$ reported the angular and temperature dependences of the effective fields in the $\mathrm{Ta}(2) / \mathrm{CoFeB}(0.8) / \mathrm{MgO}(2) / \mathrm{SiO}_{2}(3)$; we took only the value at $300 \mathrm{~K}$ and a polar angle of $4^{\circ}$, which are conditions similar to ours. They reported -4.4 and $19.42\left(\mathrm{Oe} / 10^{10} \mathrm{~A} / \mathrm{m}^{2}\right)$ for the longitudinal and transverse directions, respectively. Even though the thickness of their $\mathrm{CoFeB}$ layer is much thinner than the present study, the longitudinal field is comparable to ours, and the transverse field is larger.

Despite their remarkable works, we did not take data from the works of Fan et al. ${ }^{17}$ and Jamali et al. ${ }^{19}$ in Fig. 4 . Fan et al. ${ }^{17}$ investigated thick Py $(>2 \mathrm{~nm})$, which is out of the range of our plot. In addition, the effective field is relatively small $\left(<1 \mathrm{Oe} / 10^{10} \mathrm{~A} / \mathrm{m}^{2}\right)$ for longitudinal and transverse fields. Jamali et al. ${ }^{19}$ studied a Co $(0.2 \mathrm{~nm}) / \mathrm{Pd}(0.7 \mathrm{~nm})$ multilayer system and found huge longitudinal $\left(11.7 \mathrm{Oe} / 10^{10} \mathrm{~A} / \mathrm{m}^{2}\right)$ and transverse $\left(50.25 \mathrm{Oe} / 10^{10} \mathrm{~A} / \mathrm{m}^{2}\right)$ fields. The reason for the huge effective field in a $19.8 \mathrm{~nm}$-thick
$[\mathrm{Co} / \mathrm{Pd}]_{22}$ multilayer system is not clear. Therefore, we should omit these data sets for better readability of Fig. 4.

As shown in Fig. 4, the effective longitudinal field in the present work exhibits meaningful $1 / t_{\mathrm{FM}}$ dependence, whereas the transverse field is almost independent of $t_{\mathrm{FM}}$. Due to the limited number of data points $\left(4 t_{\mathrm{FM}}\right)$ and scattered data points, it is not quite clear why the longitudinal field is proportional to $1 / t_{\mathrm{FM}}$ in the present study. The inverse proportionality to $t_{\mathrm{FM}}$ means that the SOT effective field is purely an interface effect. Based on this argument, both effective fields must be proportional to $1 / t_{\mathrm{FM}}$ and pass through the origin. Such linear dependence is observed in the results of Fan et al. ${ }^{18}$ for a wide range of $\mathrm{Ti} / \mathrm{CoFeB} / \mathrm{Pt}$ thicknesses across the perpendicular to in-plane anisotropy regions for the longitudinal region, as shown in Fig. 4 (the blue closed circles represent $H_{L}$ ). However, the linearity of the transverse fields is not very clear (the blue open circles represent $H_{T}$ ). Kim et al. ${ }^{7}$ also reported that the transverse fields show linear dependence (green open triangles with a straight line), but the longitudinal field is not linear in the $\mathrm{Ta} / \mathrm{CoFeB} / \mathrm{MgO}$ system. However, their results have a nonzero crossing point of $1 / t_{\mathrm{FM}}$, which is physically unreasonable. One possible reason is that Kim et al. ${ }^{7}$ did not take into account the PHE contribution properly in their analysis. Quite generally, these deviations from a $1 / t_{\mathrm{FM}}$ dependence make the interfacial nature of the SOT questionable. In our case, the longit- 


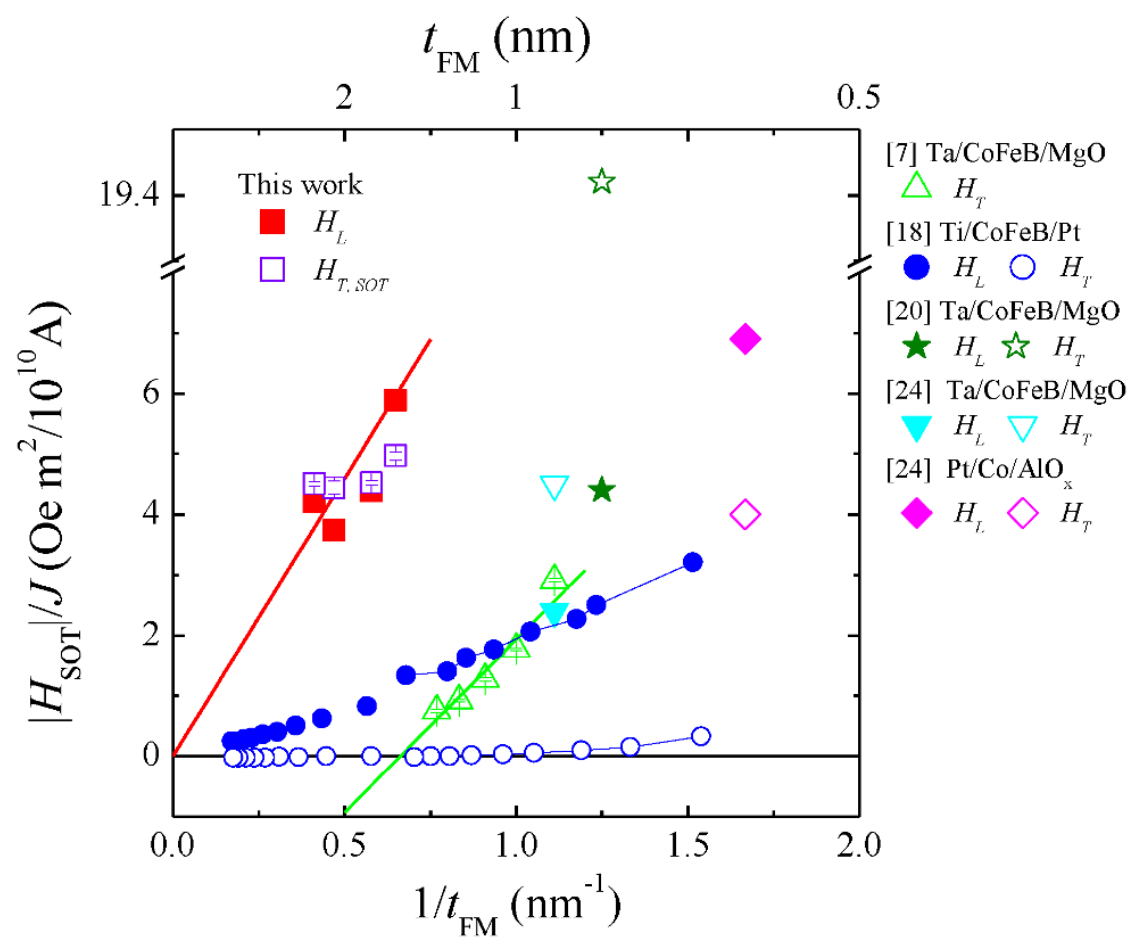

Figure $4 \mid$ SOT effective fields as a function of $1 / t_{F M}$. The absolute values of the effective longitudinal and transverse fields per $10^{10} \mathrm{~A} / \mathrm{m}^{2}$ of the present work, and the results of other groups. The all-closed (open) symbols represent the longitudinal (transverse) effective fields. The present work $(\mathrm{Pd} / \mathrm{FePd} / \mathrm{MgO})$ is represented by red and violet rectangles, the green upward triangle with the solid line is $\mathrm{Ta} / \mathrm{CoFeB} / \mathrm{MgO}$ in [7], the blue circles are $\mathrm{Ti} / \mathrm{CoFeB} / \mathrm{Pt}$ in [18], the olive starts $\mathrm{Ta} / \mathrm{CoFeB}(0.8 \mathrm{~nm}) / \mathrm{MgO}$ in [20], and the cyan downward triangles and pink diamonds are $\mathrm{Ta} / \mathrm{CoFe}(0.9 \mathrm{~nm}) / \mathrm{MgO}$ and Pt/Co (0.6 nm)/Mgo in [24]. We omitted data from [17] and [19] because of the limits of our plot range.

udinal field scales quite well with $1 / t_{\mathrm{FM}}$, with no offset. Weaker thickness dependence is observed for the transverse field, which suggests some contribution from the volume of the ferromagnet. However, the scattering of the data does not allow us to draw definitive conclusions.

Our most striking result concerns the magnitude of the effective fields (per current density). In the thickness range considered here, they are several times larger than in other reports. Assuming a $1 / t_{\mathrm{FM}}$ dependence, they remain larger than in most studies with interface PMA systems, and are only comparable with the transverse field measured by Qiu et $a .^{20}$. Our results may be compared to those of Jamali et al. ${ }^{19}$, who found larger effective fields with a $[\mathrm{Co} / \mathrm{Pd}]_{22}$ multilayered system, and ascribed the large SOT to the large asymmetric lattice distortion in the volume of their $\mathrm{Co} / \mathrm{Pd}$ multilayers. This structural asymmetry might be present in our films too, as will be discussed below.

\section{Discussion}

We now discuss possible origins of the relatively large SOT in our bulk PMA $\mathrm{Pd} / \mathrm{FePd} / \mathrm{MgO}$ system. The spin current density is given by $J_{S}\left(d_{N M}\right) / J_{S}(\infty)=1-\operatorname{sech}\left(d_{N M} / \lambda_{s f}\right)^{25}$, where $d_{N M}$ and $\lambda_{s f}$ are the thickness and the spin diffusion length of the nonmagnetic layer, respectively. Because the spin diffusion length of $\mathrm{Pd}$ is $5.5 \pm$ $0.5 \mathrm{~nm}$ at room temperature ${ }^{26-28}$, the $18.5 \mathrm{~nm}$ Pd layer is thick enough to provide saturated spin current due to the SHE. When comparing our results with previous $\mathrm{Ta} / \mathrm{CoFeB} / \mathrm{MgO}$ results $^{7}$ for a Ta thickness of $1.5 \mathrm{~nm}$ (which is thinner than or comparable to the spin diffusion length of Ta, 1.8 to $2.3 \mathrm{~nm}^{29,30}$ ), the $18.5 \mathrm{~nm}$-thick Pd layer should provide much larger spin current than that of the $1.5 \mathrm{~nm}$-thick Ta case, assuming the same $J_{S}(\infty)$. Therefore, the observed larger longitudinal effective field is reasonable if we compare this case with the experimental results for $\mathrm{Ta}(1.5 \mathrm{~nm}) / \mathrm{CoFeB} /$ $\mathrm{MgO}^{7}$.
However, our measured value does not fit theoretical models ${ }^{11,19}$. The longitudinal effective field due to the SHE per current density is given by $H_{L} / J \sim \alpha_{H} \hbar / 2 e M_{s} t_{F M} \sim 33 \alpha_{H} \mathrm{Oe} /\left(10^{10} \mathrm{~A} / \mathrm{m}^{2}\right)$, if the nonmagnetic layer is assumed to be sufficiently thick. Here, the spin Hall angle $\alpha_{H}$ is the ratio of the spin Hall conductivity and the normal conductivity of the non-magnetic layer. The value of $\alpha_{H}$ of $\mathrm{Pd}$ is known to be of the order of 0.64 to $1.2 \%$ at room temperature $e^{26,27,31-33}$. Even though there is finite uncertainty in the experimental measurement of the $\alpha_{H}$, the theoretical estimation gives $H_{L} / J \sim 0.33 \mathrm{Oe} /$ $\left(10^{10} \mathrm{~A} / \mathrm{m}^{2}\right)$, which is one order of magnitude smaller than the experimentally observed values. The simple SHE theory does not account for the observed effective field. One possible scenario is the existence of a larger spin Hall angle for Pd compared to previous reports.

The ideal FePd $\mathrm{L}_{0}$ structure shows inversion symmetry, so that only the potential gradient at the very $\mathrm{Pd} / \mathrm{FePd}$ interface should be source of Rashba effect. However, the large transverse field observed (given the weak spin-orbit coupling in Pd compared to Pt or Ta), and the weak thickness dependence of it suggest some deviation from a purely interfacial Rashba effect. Actually, the $\mathrm{L}_{0}$ structure requires a certain thickness (about 3 atomic layers) to be stabilized in the film, and the anisotropy is found to increase rapidly with the FePd thickness (see S6 in supplementary information). This means that there is an extended gradient of chemical ordering near the $\mathrm{Pd} / \mathrm{FePd}$ interface, which could be a source of potential gradient inside the FePd. This scenario is somewhat similar to the large SOT in the Co/Pd multilayer system ${ }^{19}$.

In summary, we observed large longitudinal and transverse effective fields due to the SOT in a bulk PMA Pd/FePd/MgO system. The pure longitudinal and transverse effective fields were extracted with a careful data analysis taking into account the finite PHE. The observed magnitude and thickness dependence of the effective fields suggest that the SOT do not have a purely interfacial origin. 


\section{Methods}

Sample preparations and micro-fabrication. The films were grown using molecular beam epitaxy. The stacking consisted of a $\mathrm{MgO}$ (7)/Cr (1.5)/Pd (18.5)/ $/ \mathrm{Fe}_{0.5} \mathrm{Pd}_{0.5}$ $\left(t_{\mathrm{FM}}\right) / \mathrm{MgO}(15)$ multilayer deposited on a single-crystal $\mathrm{MgO}(001)$ substrate (thicknesses in nanometers). The FePd wedge layer thickness, $t_{\mathrm{FM}}$, was varied from 1.54 to $2.43 \mathrm{~nm}$. The Pd buffer was annealed for $20 \mathrm{~min}$ at $400^{\circ} \mathrm{C}$ to obtain a flat surface. Wedge-shaped FePd was grown by co-deposition of Fe and Pd sources with a substrate temperature of $350^{\circ} \mathrm{C}$ to induce $\mathrm{L} 1_{0}$ ordering during growth with the $c$ axis normal to the plane. All layers except the FePd were deposited at room temperature. More details of the structure are explained in the previous report ${ }^{21}$ and Supplementary Information S1. The film was patterned into a hall bar using electron beam lithography and an Ar ion milling technique.

Measurement method. All the measurements were executed in a three-dimensional magnet probe station, where the magnetic field could be changed in any random direction without rotating the device. We used the harmonics technique ${ }^{21}$ to measure the current-induced effective field. The Hall voltage was measured by modulating an AC current at $307.3 \mathrm{~Hz}$ with two lock-in amplifiers: one for the in-phase first harmonics and the other for the out-of-phase ( $90^{\circ}$ off) second harmonics. We swept the magnetic field along the $x$ and $y$ directions to obtain the transverse and longitudinal effective fields, respectively.

1. Slonczewski, J. C. Current-driven excitation of magnetic multilayers. J. Magn. Magn. Mater. 159, L1-L7 (1996).

2. Katine, J. A., Albert, F. J., Buhrman, R. A., Myers, E. B. \& Ralph, D. C. Currentdriven magnetization reversal and spin-wave excitations in $\mathrm{Co} / \mathrm{Cu} / \mathrm{Co}$ pillars. Phys. Rev. Lett. 84, 3149-3152 (2000).

3. Miron, I. M. et al. Current-driven spin torque induced by the Rashba effect in a ferromagnetic metal layer. Nature Mater. 9, 230-234 (2010).

4. Miron, I. M. et al. Perpendicular switching of a single ferromagnetic layer induced by in-plane current injection. Nature 476, 189-193 (2011).

5. Miron, I. M. et al. Fast current-induced domain-wall motion controlled by the Rashba effect. Nature Mater. 10, 419-423 (2011).

6. Liu, L. et al. Spin-torque switching with the giant spin Hall effect of tantalum. Science 336, 555-558 (2012)

7. Kim, J. et al. Layer thickness dependence of the current-induced effective field vector in $\mathrm{Ta}|\mathrm{CoFeB}| \mathrm{MgO}$. Nature Mater. 12, 240-245 (2013).

8. Haazen, P. P. J. et al. Domain wall depinning governed by the spin Hall effect. Nature Mater. 12, 299-303 (2013).

9. Garello, K. et al. Ultrafast magnetization switching by spin-orbit torques. (2013) (Date of access:21/10/2013) http://arxiv.org/abs/1310.5586v1.

10. Ryu, K.-S., Thomas, L., Yang, S.-H. \& Parkin, S. Chiral spin torque at magnetic domain wall. Nature NanoTech. 8, 527 (2013).

11. Haney, P. M., Lee, H.-W., Lee, K.-J., Manchon, A. \& Stiles, M. D. Current induced torques and interfacial spin-orbit coupling: Semiclassical modeling. Phys. Rev. B 87, 174411 (2013).

12. Manchon, A. \& Zhang, S. Theory of spin torque due to spin-orbit coupling. Phys. Rev. B 79, 094422 (2009)

13. Slonczewski, J. C. Currents, torques, and polarization factors in magnetic tunnel junctions. Phys. Rev. B 71, 024411 (2005).

14. Kubota, H. et al. Quantitative measurement of voltage dependence of spintransfer torque in MgO-based magnetic tunnel junctions. Nat. Phys. 4, 37-41 (2008).

15. Sankey, J. C. et al. Measurement of the spin-transfer-torque vector in magnetic tunnel junctions. Nat. Phys. 4, 67-71 (2008).

16. Jung, M. H., Park, S., You, C.-Y. \& Yuasa, S. Bias dependences of in-plane and outof-plane spin-transfer torques in symmetric MgO-based magnetic tunnel junctions. Phys. Rev. B 81, 134419 (2010).

17. Fan, X. et al. Observation of the nonlocal spin-orbit effective field. Nat. Commun. 4, 1799 (2013)

18. Fan, X. et al. Quantifying interface and bulk contributions to spin-orbit torque in magnetic bilayers. Nat. Commun. 5, 3042 (2014).

19. Jamali, M. et al. Spin-orbit torques in Co/Pd multilayer nanowire. Phys. Rev. Lett. 111, $246602(2013)$.
20. Qiu, X. et al. Angular and temperature dependence of current induced spin-orbit effective fields in Ta/CoFeB/MgO nanowires. Sci. Rep. 4, 4491 (2014).

21. Bonell, F. et al. Large change in perpendicular magnetic anisotropy induced by an electric field in FePd ultrathin films. Appl. Phys. Lett. 98, 232510 (2011).

22. Pi, U. et al. Tilting of the spin orientation induced by Rashba effect in ferromagnetic metal layer. Appl. Phys. Lett. 97, 162507 (2010).

23. Hayashi, M., Kim, J., Yamanouchi, M. \& Ohno, H. Quantitative characterization of the spin orbit torque using harmonic Hall voltage measurements. (2013), (Date of access: 22/07/2013), http://arxiv.org/abs/1307.5603v1.

24. Garello, K. et al. Symmetry and magnitude of spin-orbit torques in ferromagnetic heterostructures. Nat. NanoTech. 8, 587-593 (2013).

25. Liu, L., Moriyama, T., Ralph, D. C. \& Buhrman, R. A. Spin-Torque Ferromagnetic Resonance Induced by the Spin Hall Effec. Phys. Rev. Lett. 106, 036601 (2011).

26. Bai, L. et al. A. Universal Method for Separating Spin Pumping from Spin Rectification Voltage of Ferromagnetic Resonance. Phys. Rev. Lett. 111, 217602 (2013).

27. Hoffmann, A. Spin Hall Effects in Metals. IEEE Trans. on Mag. 49, 5172-5193 (2013).

28. Vlaminck, V., Pearson, J. E., Bader, S. D. \& Hoffmann, A. Dependence of spin pumping spin Hall effect measurements on layer thickness and stacking order. Phys. Rev. B. 88, 064414 (2013).

29. Hahn, C. et al. Comparative measurements of inverse spin Hall and magnetoresistance in YIG-Pt and YIG-Ta. Phys. Rev. B 87, 174417 (2013).

30. Morota, M. et al. Indication of intrinsic spin Hall effect in 4 and 5 transition metals. Phys. Rev. B 83, 174405 (2011).

31. Kondou, K., Sukegawa, H., Mitani, S., Tsukagoshi, K. \& Kasai, S. Evaluation of spin Hall angle and spin diffusion length by using spin current-induced ferromagnetic resonance. Appl. Phys. Exp. 5, 073002 (2012).

32. Mosendz, O. et al. Detection and quantification of inverse spin Hall effect from spin pumping in permalloy/normal metal bilayers. Phys. Rev. B 82, 214403 (2010)

33. Ando, K. \& Saitoh, E. Inverse spin-Hall effect in palladium at room temperature J. Appl. Phys. 108, 113925 (2010).

\section{Acknowlegement}

This work was supported by National Research Foundation of Korea (grant nos. 616-2011-3-C00017, 2013R1A1A2011936, and 2012M2A2A6004261), and by the IT R\&D program of the Ministry of Knowledge Economy and KEIT (grant no. 10043398) of the government of Korea. The authors gratefully acknowledge this support.

\section{Author contributions}

H.-R.L., K.L. and Y.-H.C. carried out the harmonics measurements. M.-H.J. supervised the measurements. J.C. and C.-Y.Y. analyzed the experimental data. F.B. and Y.Sh. prepared the sample. S.M. and Y.Su. supervised the sample preparations. The manuscript was prepared and the project was designed by M.-H.J., C.-Y.Y. and Y.Su. H.-R.L. and K.L. contributed as first authors. Correspondence and requests for materials should be addressed to C.-Y.Y. or M.-H.J.

\section{Additional information}

Supplementary information accompanies this paper at http://www.nature.com/ scientificreports

Competing financial interests: The authors declare no competing financial interests.

How to cite this article: Lee, H.-R. et al. Spin-orbit torque in a bulk perpendicular magnetic anisotropy Pd/FePd/MgO system. Sci. Rep. 4, 6548; DOI:10.1038/srep06548 (2014).

This work is licensed under a Creative Commons Attribution-NonCommercialShareAlike 4.0 International License. The images or other third party material in this article are included in the article's Creative Commons license, unless indicated otherwise in the credit line; if the material is not included under the Creative Commons license, users will need to obtain permission from the license holder in order to reproduce the material. To view a copy of this license, visit http:// creativecommons.org/licenses/by-nc-sa/4.0/ 